\title{
LA DIÓCESIS DE ASTORGA, EL PADRE FLÓREZ Y LA EPIGRAFÍA MEDIEVAL
}

\author{
POR \\ Alejandro Celso García Morilla ${ }^{1}$ \\ Universidad Internacional Isabel I de Castilla
}

\begin{abstract}
RESUMEN
El estudio de la obra de los eruditos de los siglos XVI-XVIII es una de las actuales líneas de investigación del equipo del Corpus Inscriptionum Hispaniae Mediaevalium. Se trata de analizar el legado científico que estos especialistas aportaron como primera aproximación a las inscripciones medievales de España, donde es frecuente encontrar no pocos textos epigráficos. Hoy trabajamos sobre el Padre Flórez a través de una muestra concreta, aunque ciertamente representativa, como es la diócesis de Astorga, correspondiente al tomo XVI de su magna obra España Sagrada. Analizaremos su método de trabajo tratando de buscar las analogías entre éste y el actual método de la Epigrafía medieval en España. Para ello partiremos del propio concepto de inscripción que tiene nuestro erudito y del análisis de los 18 textos epigráficos que aparecen en el volumen.
\end{abstract}

PALABRAS CLAVE: epigrafía medieval; inscripción; paleografía; método epigráfico; diócesis de Astorga.

\section{THE DIOCESE OF ASTORGA, FATHER FLÓREZ AND MEDIEVAL EPIGRAPHY}

\begin{abstract}
The study of the work of the scholars of XVI-XVIII centuries is one of the current research lines of the Corpus Inscriptionum Hispaniae Mediaevalium team. This involves analyzing the scientific legacy that these specialists provided as a first approach to the medieval inscriptions of Spain, where it is common to find more than a few epigraphic texts. Today we work on Father Flórez through a particular sample, though certainly representative, as it is the diocese of Astorga, corresponding to the XVI volume of his masterpiece "España Sagrada". We will analyze its method of work trying to find the similarities with the current method of medieval Epigraphy in Spain. To do this we will start from the concept itself of inscription from our scholar and the analysis of the 18 epigraphic texts that appear in the volume.
\end{abstract}

KEY WORDS: Medieval epigraphy; inscription; paleography; epigraphic method; diocese of Astorga.

Cómo CITAR ESTE ARTículo / CITATION: García Morilla, A. C. 2017. «La diócesis de Astorga, el padre Flórez y la epigrafía medieval». Hispania Sacra 69, 139: 177-184. doi: 10.3989/hs.2017.012

Recibido/Received 05-02-2013

Aceptado/Accepted 15-09-2014

De todos es conocida la antigüedad e importancia de tan ancestral diócesis. Astorga hunde sus raíces prácticamente hasta los orígenes de la cristiandad, siendo atribuida su fundación al propio apóstol Santiago. ${ }^{2}$ Durante el periodo medieval, fueron dos los momentos de declive que sufrió la sede episcopal. En primer lugar, la despoblación de Alfonso I, que provocó el abandono de la sede. En segundo, los conflictos acaecidos a consecuencia de la doctrina milenarista, que llevaron a la quema de monasterios, documentación y enajenaciones de propiedades eclesiásticas.

1 alejandro.garcia@ui1.es; agarm@unileon.es / ORCID iD: http:// orcid.org/0000-0002-2717-8285

2 Quintana Prieto 1972.
Sin embargo y a pesar de los conflictos jurisdiccionales que se fueron sucediendo durante el periodo altomedieval con las recién creadas diócesis de León, Palencia, Zamora u Orense, Astorga ha sido uno de los principales centros episcopales de la península, con casi un centenar de monasterios dependientes, con un abanico cronológico tan amplio como sus estilos arquitectónicos, que reflejan la pujanza de la Diócesis durante siglos.

Por su parte, el Padre Flórez es uno de esos eruditos nacionales que no necesitan presentación. Nacido en Villadiego (Burgos) en 1792, ingresó en la Orden agustiniana en 1718. Estudió Artes y Teología en Valladolid y Salamanca respectivamente y se doctoró en 1729 en Alcalá de Henares. ${ }^{3}$

\footnotetext{
3 Peset 2006: 113.
} 
Conocido habitualmente por su faceta de historiador, este agustino burgalés también destacó como paleógrafo y epigrafista, como veremos a continuación. Los diferentes estudios llevados acabo sobre la figura del Padre Flórez llevan a considerarlo como una persona de elevada vocación religiosa, como un agustino de elevada categoría humana pero, sobre todo, como un intelectual de primera fila. ${ }^{4}$

El trabajo que presentamos a continuación pretende vincular nuestros estudios de Epigrafía medieval con la obra de este gran historiador o erudito del siglo XVIII, como punto de partida de una ciencia aún joven y novedosa..$^{5} \mathrm{Y}$ esta relación pretendemos hacerla a través de la diócesis de Astorga: será una muestra concreta de la metodología de trabajo de Flórez, que a su vez pondremos en conexión con el método empleado para la edición del Corpus Inscriptionum Hispaniae Mediaevalium. ${ }^{6}$

\section{LA ESPAÑA SAGRADA}

No pretendemos, ni podemos, hacer aquí un análisis pormenorizado de esta magna obra. Sin embargo, sí parece necesario trazar unas breves pinceladas sobre ella a modo de introducción. ${ }^{7}$

Bien es sabido que la España Sagrada fue concebida como una recopilación histórica de la geografía eclesiástica española. Aunque el principal empeño de Flórez fue la recuperación de las fuentes documentales como base firme para una recreación fidedigna de los hechos históricos, ${ }^{8}$ resultó ser un compendio de enorme valor no sólo por el conjunto de documentos, sino también del resto de fuentes narrativas y epigráficas, que le aportan infinidad de noticias e ilustraciones que pretendían esclarecer los hechos históricos de la Iglesia española. La obra se iniciaba en 1747 con la aparición de los dos primeros volúmenes. ${ }^{9}$ El planteamiento varió desde sus orígenes; al principio la obra tenía un cáliz mucho más genérico, pero a partir del volumen quinto, el Padre Flórez comienza a prestar atención a los aspectos locales y más concretos. Según los expertos, es justamente a partir del tomo XVI, dedicado a la iglesia de Astorga, cuando esta tendencia localista se acentúa. ${ }^{10}$

${ }^{4}$ La figura del P. Flórez ha sido sistemáticamente estudiada desde hace años. Sin embargo, con motivo del tercer centenario de su nacimiento, el ayuntamiento de Villadiego -su ciudad natal- promovió un congreso internacional que culminó en un magnífico volumen al que remitimos para un conocimiento más exhaustivo de la vida y obra de este célebre burgalés. Cf, AA. VV. 2006.

5 Así se expresaba el profesor Favreau el año 1979 «L'épigraphie médiévale est encore trop jeune pour qu'on puisse prétendre en donner un tableau méthodique général». Cf. Favreau 1979.

6 El Corpus Inscriptionum Hispaniae Mediaevalium es un proyecto de investigación del Ministerio de Ciencia e Innovación, bajo la dirección del prof. Dr. D. Vicente García Lobo que tiene por objeto la publicación, provincia a provincia, de todas las inscripciones medievales de España; equipo de investigación al que pertenecemos desde al año 2009.

7 Son muchos los trabajos que hay sobre esta obra del Padre Flórez. Nosotros remitimos, con carácter general, a Campos y Fernández de Sevilla 2000.

8 Vid. con carácter general Fernández Flórez 2006.

9 Flórez 1747.

10 Borreguero Beltrán 2006: 11.

\section{LA IGLESIA DE ASTORGA. LAS FUENTES}

Como decíamos anteriormente, el tomo XVI de la España Sagrada es el dedicado a la diócesis de Astorga. El volumen de documentación recuperada para esta diócesis fue motivo suficiente para dedicarle un solo tomo. ${ }^{11}$ Por lo que a nosotros se refiere recoge un total de dieciocho inscripciones: ocho Epitaphia sepulcralia, nueve Consecrationes, y un Monumentum restaurationis; este es el material con que vamos a trabajar.

Estas inscripciones son consideradas como fuente de primer orden para el conocimiento de la diócesis y sus datos son tomados como testimonios fidedignos para la reconstrucción de su historia. La actividad publicitaria ${ }^{12}$ -eso son las inscripciones: publicidad- resultaba costosa y respondía a inquietudes muy concretas de sectores sociales limitados. Por tanto, el estudio que aquí presentamos nos va a permitir conocer algunas de esas vicisitudes de la Iglesia asturicense aunque bajo el prisma de nuestro erudito agustino.

Sin embargo, creó, el punto de partida en un estudio de estas características es saber qué entiende nuestro autor por inscripción. Desde el tomo que hoy analizamos no resulta fácil extraer una definición exacta, aunque sí podemos descubrir qué espera de la inscripción. De ella espera que perpetúe lo que allí se dice, como vemos a propósito del epitafio del abad Esteban: «en el Sepulcro de otro Santo Varon, Ilamado Estevan, cuya memoria ha quedado perpetuada en la Inscripción siguiente». ${ }^{13}$ La define como "memoria" y qué duda cabe de que esta memoria no es otra cosa que la publicidad universal y permanente de un hecho. ${ }^{14}$ Por tanto, la considera como una fuente de información al uso; como un recurso más para la reconstrucción fidedigna de la historia. Queda probada la valoración y uso que hace de las inscripciones cuando edita los textos, los analiza críticamente y utiliza sus datos para esta recreación histórica del pasado de la diócesis.

Por otro lado, hay que tener en cuenta que Flórez, como buen historiador, cuenta con un método de trabajo sistemático como base científica de cuanto dice. Este método tiene tres fases bien definidas: estudio bibliográfico, trabajo de campo y trabajo o labor de gabinete. Sin embargo no es igual de profundo en todos los procesos. Para la fase bibliográfica empleó un buen número de fuentes y referencias de autores anteriores que estimaba de reconocido prestigio; de hecho, buena parte de las noticias que nos traslada son tomadas directamente de éstos, aunque no suele faltar la referencia de dónde es tomada la información: "La pondrémos aqui como la dió Morales lib. 15. Cap. 23.». ${ }^{15}$ No es menos cierto que esas noticias que recoge son sometidas a crítica histórica y paleográfica y que en numerosas ocasiones acierta en corregir lecturas erróneas como veremos más adelante.

\footnotetext{
11 Así lo señala el propio Flórez en la presentación del tomo. Cf. Flórez 1762.

12 Sobre la importancia de las inscripciones como medio de comunicación publicitaria viene hablando el prof. García Lobo desde el año 1991. Cf. García Lobo 1991.

13 Flórez 1762: 41

14 Así viene definiendo García Lobo a las inscripciones medievales desde 1991: «cualquier testimonio escrito en orden a una publicidad universal y permanente» Cf. García Lobo 1991: 17.

15 Flórez 1762: 128.
} 
Para el trabajo de campo le fue fundamental la ayuda de otros informadores. Al igual que otros autores, como Morales, ${ }^{16}$ Flórez contó con estos colaboradores en los diferentes centros para informar sobre el contenido y vicisitudes de las inscripciones: «recogió y me comunicó la copia el Rmo. Alonso» ${ }^{17}$ o "Pero la diligencia del docto Cisterciense Alonso, ha descubierto el sepulcro en Carracedo, en un nicho (...) con Inscripcion por la parte de la cabeza». ${ }^{18}$

También resulta peculiar en Flórez la forma de edición de las inscripciones. Siempre que ve los epígrafes o que los considera de especial importancia reproduce el texto dibujando sus caracteres lo más fielmente posible. En estos casos, no es raro que acompañe el texto de la transcripción paleográfica que le sirve para verificar o corregir lecturas anteriores. No sucede lo mismo cuando la inscripción es tomada de otros o cuando considera que el texto no tiene especial dificultad; entonces se limita a reproducir la transcripción.

Pero cabe preguntarse en qué medida el método epigráfico de Flórez recoge la información epigráfica que en la actualidad le pedimos a nuestros estudios. Eso será lo que hagamos: relacionar su información con nuestro actual método de trabajo para ver si responde a las preguntas que hoy le hacemos a las inscripciones medievales. Por ello, pasamos a desglosar los capítulos o partes en que dividimos el estudio epigráfico hoy en día.

\section{GÉNESIS: AUTOR, DESTINATARIO Y ROGATARIO}

El estudio del proceso de génesis de las inscripciones es el punto de partida para un análisis epigráfico moderno. ${ }^{19}$ Se trata de rastrear, a través de las huellas dejadas en las inscripciones, cómo fue su proceso de elaboración. Desde hace algún tiempo, venimos diciendo que el proceso de génesis de las inscripciones es uno de los menos conocidos ${ }^{20}$ y más difícil aún nos resulta saber si algunas de estas huellas fueron analizadas por Flórez; parece que sí.

Como es lógico, no se trata de alusiones directas a nuestro método, pero sí que se nos permite establecer ciertas analogías a través de los comentarios que acompañan a cada inscripción. Así, por ejemplo, lo vemos en los datos proporcionados a propósito del epitafio del abad Esteban de Santiago de Peñalba, donde dice: "Cerrados en estas reclusiones los hombres, de quienes el mundo no era digno, tenian toda su alama en el Cielo (...) Algunos la tuvieron preciosa aun para los ojos de los hombre, dejando señalados sus sepulcros para egemplo y refugio de los demas». ${ }^{21}$ Aquí señala quien fue el autor de la inscripción. El propio abad tuvo intención de perpetuar su memoria escribiendo-o mandando escribir-su epitafio. También recoge la noticia

16 Estas referencias en la obra de Morales son muy habituales: «nombrare tambien algunas vezes a fray Alonso Chacon, Rector del colegio de santo Thomas de Sevilla: porque la gran noticia que tiene de harto de la antiguedad de España, es cosa muy señalada en la mucha otra doctrina que en Theologia tiene. Y de los mucho que el ha descubierto en algunas antiguedades, me comunico buena parte». Cf. Rodríguez Suárez 2010.

17 Flórez 1762: 196.

18 Ibídem: 209.

19 A este respecto seguimos los dictados expuestos por García Lobo y Martín López (1995) y desarrollados por García Lobo (2001).

20 En estos términos se expresaba la Dra. Martín López a propósito de su trabajo sobre los centros scriptorios epigráficos en Palencia. Cf. Martín López 2007: 203.

21 Cf. Flórez 1762: 40-41. de la voluntad del obispo don Nuño de querer enterrarse en la iglesia de San Dictino como reza su epitafio. ${ }^{22}$ Además de esta voluntad advierte que: «Este Prelado se llamaba Nonno, Obispo de la misma Iglesia de Astorga, que dictó el Epitaphio en vida y por tanto no señaló el año ni dia de su muerte». ${ }^{23}$ Se trata de la alusión más directa que tenemos al autor de una inscripción y que presenta como muestra de lo que debía ser costumbre. Lo mismo sucede con el epitafio del obispo Ordoño, donde el estilo directo del texto hace pensar a Flórez que estamos ante el autor: «....La inscripción de su sepulcro, donde celebrando su vida, manda que no lloren la muerte; pues aunque el cuerpo yace allí, la alma subió alegre à los Cielos». ${ }^{24}$ En otras ocasiones, a pesar de que la referencia al autor en el texto epigráfico es explícita, Flórez no hace mención alguna al dato, como sucede en la Consecratio de la iglesia de Santa María de Valverde donde al final del texto leemos: PS. M. FIERI PRECEPIT (Ap. n. 11). Tampoco reflexiona a lo largo de este tomo sobre la figura del destinatario de la inscripción ni sobre lo que nosotros llamamos rogatario de la misma o autor material, a pesar de que en algunos casos sí se hace alusión directa a él en las inscripciones.

\section{FORMA: CARACTERES EXTERNOS, INTERNOS Y FUNCIONALES}

Al igual que sucediera en el apartado anterior, Flórez analiza aisladamente alguno de estos aspectos que nos resultan de especial importancia para la crítica epigráfica. Un buen ejemplo es cómo respeta los caracteres de las inscripciones cuando él mismo las ve y las transcribe. Como reflejamos en el Apéndice, reproduce las formas de las letras y señala las peculiaridades que pueda encontrar; especialmente si éstas han supuesto algún problema de interpretación o lectura para otros autores. Así en la Consecratio de la ermita de la Santa Cruz en San Pedro de Montes reflexiona sobre los problemas de datación que han tenido otros autores debidos a una mala interpretación de los numerales y de letra $\mathrm{D}$ de Decembris que otros habían leído como una O para Octobris. De la misma manera procede al corregir el numeral $\mathrm{XL}$ de la data, advirtiendo que algunos de los autores anteriores «despreciaron la vírgula que hace XL. al X.» ${ }^{25}$; así la data no concordaba con el gobierno de san Genadio en San Pedro de Montes. En la misma línea reflexiona sobre el error cometido por Sandoval en la Consecratio de la iglesia de San Adrián que Yepes corrige (Ap. n. 7); y lo mismo sucede en el Epitaphium sepulcrale del obispo Ordoño (Ap. n. 8) advirtiendo sobre error de lectura de autores anteriores para los días en que gobernó la sede -19 y no 18-, la "prima feria", del die VII Kal. Martii que transcriben como Domingo siendo en verdad Miércoles y el final de la inscripción que no termina con «requiscat, sino con requiescit in pace». ${ }^{26}$

Otro aspecto que refleja la meticulosidad de nuestro autor es que advierte de las limitaciones de la edición para reproducir fielmente los epígrafes. Así, en varias ocasiones señala que los puntos de separación de palabra son tres en lugar de los dos que figuran en la imagen. También presta atención al conjunto de caracteres externos; esto es, la escritura, abreviaturas, signos especiales, etc. A propósito de la Consecratio de la iglesia de

\footnotetext{
22 Ibídem: 83-84

23 Ibídem: 84

24 Ibídem: 181.

25 Ibídem: 136.

26 Ibídem: 182.
} 
Corullón (Ap. n. 9) dice: «El carácter es como en otras de aquel tiempo, con algunos enlaces de letras...». ${ }^{27}$ Más exhaustivo es al analizar las peculiaridades gráficas del Epitaphium sepulcrale del obispo Gimeno (Ap. n. 13): «Ponemosla en su proprio carácter, por algunas particularidades. La V. todas son como A. àcia arriba con la línea del medio». ${ }^{28}$

También analiza la singularidad que presenta el epitafio del abad Florencio de Carracedo desarrollado en tres cuerpos en torno a una cruz y un báculo abacial: «en cuya lapida grabaron la Cruz y el Baculo Abacial, con Inscripcion por la parte de la cabeza, y al brazo izquierdo de la Cruz de arriba abajo, formando tres inscripciones ${ }^{29}$ y edita el texto respetando los elementos decorativos que debieron realizarse con anterioridad al epígrafe (Ap. n. 14).

Desde el punto de vista de los aspectos que configuran internamente el epígrafe, o, como los llamamos actualmente, caracteres internos de las inscripciones, se limita a tres de nuestras actuales tipologías: Consecrationes, Funera y Monumenta. Estas tipologías se establecen en función del verbo notificativo de la inscripción. ${ }^{30}$ Lógicamente, Flórez no utiliza esta nomenclatura haciendo alusión únicamente a epitafios, lápidas de consagración e inscripciones. Esta limitación tipológica responde al propio contenido de la obra que versa sobre la antigua Iglesia de Astorga, donde los datos recogidos más relevantes son las fundaciones de iglesias, monasterios y catedrales y el episcopologio de la diócesis.

Lo más significativo dentro de este apartado es la advertencia -indirecta- que hace sobre la circulación de modelos o formularios ${ }^{31}$ cuando ve repetidas ciertas expresiones estereotipadas en las Consecrationes de la iglesia de San Martín de Pieros (Ap. n. 10) y la de Santa Marina de Valverde (Ap. n. 11) que comienzan «Ecce domus Domini et porte caeli, aeclesia difusa et non divisa». ${ }^{32}$

Respecto al análisis del estilo de las inscripciones resulta especialmente sugestivo el caso del epitafio de Gimeno III (Ap. n. 13). Flórez confirma que se trata de este obispo por el verso leonino que presenta la inscripción. Tras este Gimeno no hubo más prelados de este nombre y no se puede corresponder con algún otro anterior pues en ese tiempo no se utilizaba ese tipo de verso. ${ }^{33}$

Mucho menos minucioso es al tratar sobre temas relacionados con la funcionalidad del epígrafe y los aspectos que lo configuran como medio de comunicación publicitaria universal y permanente: emplazamiento, legibilidad y perdurabilidad. ${ }^{34}$ Únicamente nos da la noticia del traslado de una de las inscripciones. Se trata del epitafio del obispo Gimeno antes citado (Ap. n. 13): «...según el Epitafio que se conserva en una lapida sacada de la Iglesia de Santa Marta, y existente en casa de D. Pedro Arguellos, calle de la Rua, la qual dice:».. ${ }^{35}$

27 Ibídem: 190.

28 Ibídem: 204.

29 Ibídem: 209.

30 Cf. García Lobo y Martín López 1995: 35-39.

31 Estas repeticiones de fórmulas en las inscripciones son las que posibilitan la clasificación de los epígrafes en relación a su función. Su estudio nos permite hablar de inscripciones librarias e inscripciones diplomáticas y dentro de ellas podemos establecer "tipos" en función del verbo notificativo. Cf. Nota anterior.

32 Flórez 1762: 190-191.

33 Ibídem: 205.

34 García Lobo y Martín López 1995: 40.

35 Flórez 1762: 204.

\section{TRADICIÓN EPIGRÁFICA Y CONSERVACIÓN}

Es otro de los aspectos que en la actualidad venimos trabajando en Epigrafía medieval. Entendemos por tradición epigráfica "la forma de llegar a nosotros los textos de las inscripciones». ${ }^{36}$ Según esto, las inscripciones puede ser: originales, copias o falsos. Flórez aquí también responde a nuestras expectativas cuando nos da noticia de una inscripción realizada tiempo después de los hechos que narra, o lo que nosotros llamaríamos un original tardío: «Según expresa la Inscripcion perpetuada en una piedra de aquella Santa Casa... Este monumento, aunque no sea del tiempo de san Genadio, fue puesto en su monasterio por memorias proprias de la Casa, y como útil para algunas materias lo reproducimos». ${ }^{37}$ Aquí observamos como hace crítica de los datos históricos del texto epigráfico que considera cierto; no duda de la veracidad de los hechos a pesar de que el análisis paleográfico sitúa la inscripción en época más moderna.

También se trataría de una inscripción realizada tiempo después de los hechos que narra la Consecratio del altar e iglesia del monasterio de Sahagún. Aunque señala que el texto es tomado de Argáiz, sostiene que se trata de una memoria realizada tiempo después porque no tiene Era, ${ }^{38}$ lo habitual para la datación de estos epígrafes del siglo XII. Esta idea vendría apoyada por una segunda inscripción que se conserva en la iglesia, donde además de la noticia de la consagración de la iglesia se menciona la sepultura del obispo Fernando, cuyo óbito no pudo ser antes de 1189 cuando figura el último privilegio por él firmado.

Es especialmente interesante la edición de Flórez cuando recoge la reproducción facsímil que hemos dado en llamar "copias imitativas". Suponen una de las mayores aportaciones para la Epigrafía medieval por recuperar textos epigráficos susceptibles de ser analizados tanto en sus caracteres internos como en los externos.

Respecto a la conservación también contamos con algunos datos recogidos por Flórez. Sobre la recuperación de la Consecratio de Santiago de Pañalba (Ap. n. 12) dice los siguiente: "Según consta por Inscripcion conservada en dicha Iglesia, pero hasta hoy no conocida; à causa de que blanqueando la Iglesia por adentro, se decuidó el Albañil en cubrir las letras: pero descubiertas en nuestros días... ${ }^{39}$

Finalmente hemos de concluir señalando que todo lo dicho anteriormente no puede ser entendido como un atisbo de método epigráfico, sino que es la consecuencia lógica de un riguroso y sistemático análisis histórico donde las inscripciones han sido consideradas como fuente de primer orden y por ello han sido tratadas desde un punto de vista crítico y ciertamente científico. Gracias a ello podemos establecer paralelos con nuestro método actual, ya que en su concienzuda labora no descuidó aspectos tales como el análisis del soporte, la escritura y sus vicisitudes, el texto y su estilo, etc. Aspectos estos que conforman buena parte de las inquietudes científicas de la crítica epigráfica moderna. Su obra supone un inicio, una reflexión y, desde luego, un punto de partida para el estudioso de la inscripciones medievales.

\footnotetext{
36 García Lobo y Martín López 1995: 41.

37 Flórez 1762: 131-132.

38 Ibídem: 220.

39 Ibídem: 196.
} 
APÉNDICE

-1 -

1132

Epitaphium sepulcrale del abad Esteban.

A. SANTIAGO DE PEÑALBA, Monasterio.

PUBL: Flórez, España Sagrada, XVI, p. 41.

a. Stephanus famulus Dei Franco, quando migravit de hoc seculo. Orate pro eo.

b.

Clauditur in Cbristo sub marmore Stepbanus isto
Abbas egregius moribus eximius
Dir Domini verus, rectusque tenore severus,
Discretus, sapiens, sobrius, ac patiens.
Grandis bonestatis, magneque eir pietatis,
Dum sibi posse fuit, vivere dum licuit.
Quem nobis clarum genuit gens Francigenarum,
Rectorem juvenum, dogma, decusque fenum.
Gervasij festo cessit, fragilique senecta.
Virtas celsa Dei propitietur ei.
'Annum centesimum duc, septies addito denum.
Mille quibus socies, qua fuit Era scies.
XIII. Klds. Ful. obiit Stepbanus, Era MCLXX.
Pelagius Fernandex jussit fuerit, Petrusq. notavit.

- 2 -

Epitaphium sepulcrale de cierto Viviano del monasterio en el San Pedro.

A. SAN PEDRO DE MONTES, Monasterio, piedra cuadrada de cuatro renglones. (Él lo reproduce en el doble de líneas por la edición).

PUBL: Flórez, España Sagrada, XVI, pp. 61-62.

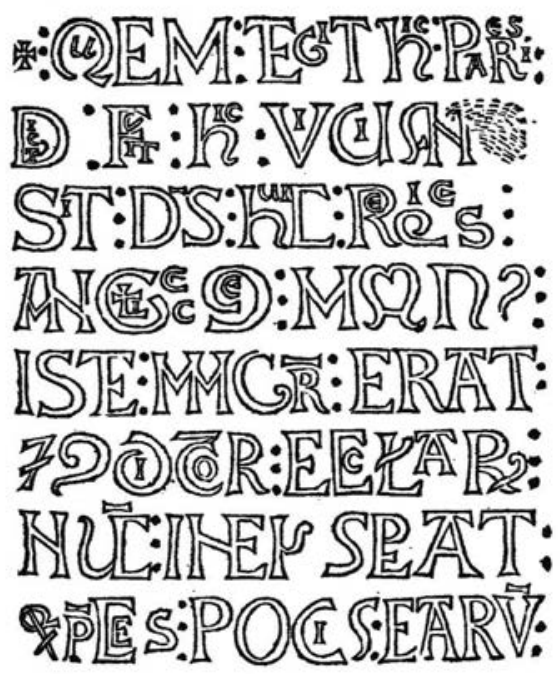

Quem tegit hic paries diatus fuit bic Vivianms. Sit Dews huic requies, Angeliceque manus. Iste Magister erat, $\sigma$ conditor Ecclesiarume. Nunc in eis sperat, qui preces poscit (*) earwm.

$-3-$

S. IX

Epitaphium sepulcrale del Obispo Nuño.

A. ASTORGA, Iglesia de San Dictino o Dictinio.

B. Morales, libr. II, Cap. V. Fol. 10; Flórez, España Sagrada, XVI, p. 83.
IN NOMINE DOMINI NostRI JESU CHRISTI INTRO HOC TUMULUM REQUIESCIT FAMULUS DeI NONNUS EPISCOPUS. REQUIEVIT IN PACE SUB DIE

Si QUIS EPISCOPUS R. PRAECESSOR VEL ACTOR CUJUSQUE VASUM ISTUM IN QUO JACEMUS, AUT CORPUSCOLUM NOSTRUM AB HINC TOLLERE, AUT COMMOVERE VOLUERIT, ANAthema sit, et ante Tribunal Christi SANCTO DICTINIO EPISCOPO ET Confessore suo, cujus nos parietrbus MANU SUA FACTIS VEL UMBRACULIS TEGIMUR, JUditio contendat: et Datan et Abiron, quos terra vivos absorbuit, PARTEM RECIPIAT, ET CUM JUDA TRADITORE SORTIATUR ET TENDAT: AC TREMENDO JUDITU DIE NON EVADAT ET STRIDORE DENTIUM.

$-4-$

893

Consecratio del Monasterio de Valdediós.

A. OVIEDO, Monasterio de Valdediós.

B. Morales, Libr. 15, Cap. 23; Flórez, España Sagrada, XVI, p. 128.

CONSECRATUM EST TEMPLUM HOC AB EPISCOPIS SEPTEM $M_{n}$, RUDESINDO. DUMIENSE, NAUSTI CONIMBRIENSE, SISNANDO IRIENSE, RANULPHO ASTORICENSE, ARGIMIRO LAMECENSE, RECCAREDO LUCENSE, ELLECANA CESAR AUGUSTANENSE, SUB ERA NONGENTESIMA TRIGESIMA PRIMA, DIE DECIMO SEXTO KAL. OCTOBRIS.

$-5-$

895

Monumentum restaurationis del monasterio de San Pedro de Montes.

A. SAN PEDRO DE MONTES, Monasterio, piedra conservada en la casa, inscripción perpetuada en piedra.

PUBL: Flórez, España Sagrada, XVI, p. 131.

INSIGNE MERITIS BEATUS FRUCTUOSU;, POSTOUAM COMPLUIENSE CONDIDIT COENOBIUM, NOMINE SANCTI PETRI BREVI OPERE IN HOC LOCO FECIT ORATORIUM. POST QUEM NON IMPAR MERITIS VALERIUS SANCTUS OPERE ECCLESIÆ DILATAVIT. NOVISSIME GENNADIUS PRESBYTER CUM DUODECIM FRATRIBUS RESTAURAVIT ERA DCCCCXXXIII. PONTIFEX EFFECTUS , A FUNDAMENTIS MIRIFICE, UT CERNITUR DENUO EREXIT, NON OPPRESIONE VULGI, SED LARGITATE PRETII, ET SUDORE FRATRUM HUJUS MONASTERII. CONSECRATUM EST HOC TEMPLUM AB EPISCOPIS OUATUOR GENNADIO ASTORICENSE, SABARICO DUMIENSE, FRUNIMIO LEGIONENSE, ET DULCIDIO SALMANTICENSE, SUB ERA NOVIES CENTENA, DECIES QUIINA. TERNA ET QUATERNA, NONO KAL.NOVEMB. 
$-6-$

905

Consecratio de la ermita de la Santa Cruz.

A. SAN PEDRO DE MONTES, ermita de la Santa Cruz.

B. SAN PEDRO DE MONTES, ermita de la Santa Cruz (nueva ermita del S. XVIII).

PUBL: Domingo de Ibarreta; Flórez, España Sagrada, XVI, p. 137.

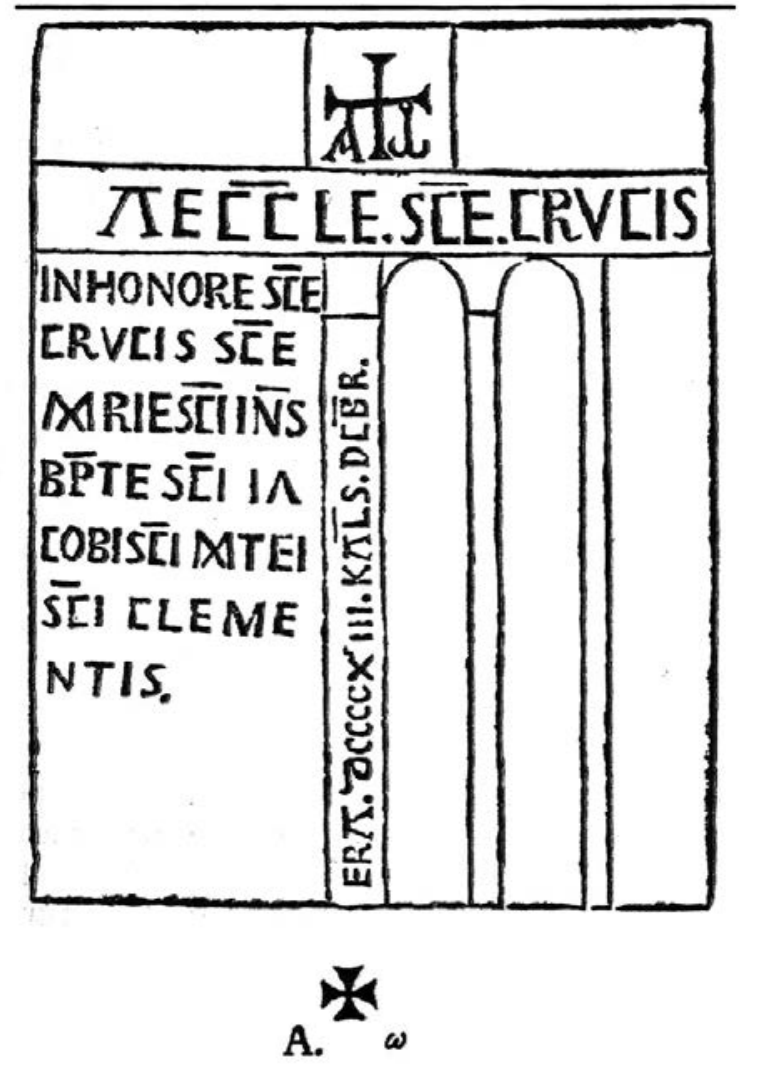

AECLESIE SANCTE CRVCIS. IN HONORE SANCTE CRVCIS. SANCTE MARIE. SANCTI IOANNIS BAPTISTE. SANCTI IACOBI. SANCTI MATEI. SANCTI CLEMENTIS. ERA DCCCCXIIII. KAL.DECEMBRIS.

-7 -

920

Consecratio de la iglesia de San Adrián de Boñar.

A. BOÑAR, Iglesia de San Adrián de Boñar, piedra conservada.

PUBL: Sandoval, Yepes, IV, fol. 357; Flórez, España Sagrada, XVI, p. 148.

\section{CONSEGRATUM EST TEMPLUM AB} EPISCOPIS FRUNIMI, CIXILA , ET FORTIS, ERA ICCCCLVIII. IV.IDUSS OCT.

\section{-8 -}

1065

Epitaphium sepulcrale del obispo Ordoño.

A. ASTORGA, Parroquia de Santa Marta, sepulcro.

PUBL: Flórez, España Sagrada, XVI, p. 182.
Tolle precor lacrimas, cessent sospiria lector. Non jacer in tumnlo res lacrimanda diu.

Hic raptus recubat felici sorte sacerdos, Quem lextum coelis intulit alma fides.

Ordonius cui nomen erat, sed Episcopus, alta Doctrina pollens, virginitate nitens:

Corde pius, vulku placidus, \& mente benignus, Prudenter simplex, simplicitate sapiens.

Omnibus in studris tantum celebratus, ut illi Cederet eloquio Roma discrta suo.

Non aliquem verbo, non facto læsit iniquo : Cum bonitate pius, cum pietate bonus, Non qui multiplices auri congessit acervos, Sed dando miseris, largus ubique fuit. Ut breviter dicam tenuit sic corpore mundum, Ut corde atque animo cerncret ille Deum. In Episcopatus degens vitam fere ankos tres, or diebus XVIIII. obiit ....... hora tertia, Era centesima tertia post millena, die VII. Kal. Martij. Anima ejus requiescit in pace.

-9 -

1086

Consecratio de la iglesia de Corullón.

A. CORULLÓN (Villafranca): Iglesia, hueco del pórtico: (donde pone dos puntos son tres).

PUBL: Flórez, España Sagrada, XVI, p. 190.

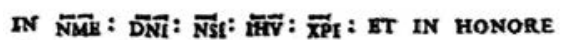

SCT: STEPHANI: SACRATYS: EST: LOCVS ISTR : AB $\overline{\text { EPO }}$ ASTORIC हีSE: TMME: OSMVNDO : IN ERA : CENTIES : DENA : ET : BIS: QYINQYAGENA : ATQYE : DVO: DFNA

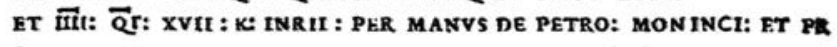
CVIS : ORIGO : RHAT: POSTEA : AD ANNOS : VII : FIECIT: EAM: ET A FVNDAMENTO: CONSTR VXIT: ET IN ALTOS VII: FVIT: PR FECTA

\section{$-10-$}

1086

Consecratio de la iglesia de San Martín.

A. PIEROS (Villafranca), Iglesia de San Martín, pared de la sacristía. (Donde pone dos puntos de separación, son tres).

PUBL: Flórez, España Sagrada, XVI, pp. 190-191.

A ECCE: DOMUS: DOMINT: ET:PORTE: CELI: ECCLESIA: DIFUSA:ET:NONE DIVISA: IN: HONOREM: SANCTI: MARTINI: RPISCOPI: ET: CONFESORIS: S:SALVATORIS:CUM:XII:APOSTOLIS:ET:SANCTE:MARIE:VIRGINIS:ET ALIORV PLURIMORUM:SANCTORUMEMARTIRUM: CONPESORUAE ATQUE:VIRGINUME: FT: EDIFICAVIT: PETRUS: PRE SBITER: IPSA: ECCLESLA: ET ALVARUS:

CARSEA: ET: UXOR: SCA: ADOSINDA: ET: RODERIGUS: PRESBITER: COMFLEVIT : EAM: ET: ORNAVIT: OMNA: BONA: QUE: IBI: EST: INTUS: ET: FORIS: IN DIEBUS: ADEFONSUS: REX: REGNANTE: IN: LEGIONE: ET: IN: TOLETUM: ET: CONSECRAVIT: EAM:OSMUNDUS: EPISCOPUS: ASTORI CENSE: SEDIS: SUB: ERA: CXXIH:POST: M:QUOTUM:XIII:KAL: DECEMB.

\section{-11 -}

991

Consecratio de la iglesia Santa Marina.

A. VALVERDE, Iglesia de Santa Marina.

PUBL: Yepes, Crónica, V, p. 227; Flórez, España Sagrada, XVI, p. 190.

ECCE DOMS DNI ET PORTE CAELI : AECLESIA DIFUSA. ET NON DIVISA GENETRICIS DNI SCE MARIE IN ONORE IPSIUS.DOMNUS VIRMUNDUS REX IN ERA XXVIIIIa PS. M. FIERI PRECEPIT. 
-12 -

\section{5}

Consecratio de la iglesia de Santiago.

A. PEÑALBA, Iglesia de Santiago, inscripción inédita por haber estado encalada hasta ese momento. Recogida por el Rmo. Alonso que le da la noticia a Flórez. Advierte que el texto epigráfico está en caracteres modernos.

PUBL: Flórez, España Sagrada, XVI, p. 196.

IN ERA CX $x^{\wedge}$ III. $P^{s}$ MLE ET VII. ID $\bar{M} \bar{R} C$ CONSECRATA EST HEC $\overline{\text { ECLA }}$

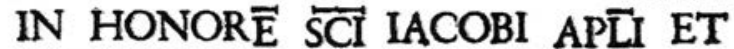
DIV PLMOR

\section{Esto es : In Era centesima qua- dragesime tertia post milesimam, ๘VII. Idtus Marcij consecrata est hac Ecclesia in hanorem Sancti facobi Apostoli, $\sigma$ divo- rum plurimorum. $Y$ aunque}

\section{- 13 -}

1141

Epitaphium sepulcrale del obispo Gimeno III.

A. ASTORGA, Iglesia de Santa Marta.

B. AStORgA, Casa de D. Pedro Arguellos, c/ de la Rua, Lápida, copia la grafía que tuvo y la transcribe paleográficamente.

PUBL: Flórez, España Sagrada, XVI, p. 204.

\section{Presul xemenus DROBIZATIS LVCE SERENYS 2VIIACEZ hOC ZVMVLO GRAGHS ERAG POPYLO CONSZAMS DISCREZVS LARGGYS YIVSAGZT FACEGYS CLAKVS STKOGEIE JRESIDIUM SAZRIG L $\forall$ CEAG IN CELIS fSRECIBVS SCI MICAhELLIS C $\forall I \forall S ~ L \forall C E ~ K \forall I T G$ AKसA2 DESEK KIG}

\section{Presul Xemenus probitatis lace serenus, qui jacet boc tunulo, gratus erat populo, constans, discretus, largus, pius, atque facetus. Clarus progenie, presi- dium patrie. Luceat in Celis pre- cibus Sancti Michaellis, cujus luce ruit, arvaque deseruit.}

$-14-$

1160

Epitaphium sepulcrale del abad Florencio.

A. CARRACEDO, Monasterio, nicho del Capítulo, triple inscripción con cruz y báculo abacial.

PUBL: Noticia del cisterciense Alonso; Flórez, España Sagrada, XVI, p. 209.

a. ERA M.C. NONAGESIMA. VIII KIS. IANV.ARII: OBITT FLORENCTVS ABBAS QVI REXIT ECCLESIAM ANNIS XIV

b. PLORYTT HIC DIGNVS FLOREN
CTVS ABBA BENIGNVS

C. CLAVDITVR.IS.XPO. TVMVLO FTORENCTYS RSTOAABAASPOPVLISQYE PER OMNLA CARVS. CARME MANENS FRLGULI DNI6 PRELATVS OVILK, FLORE PVDICITIE VEREQYE VALORE SAPYENCIB. FLORENS FLOREBAT DICTIS FACIISQ, VALEBAT, MOR BBY W' VIXIT

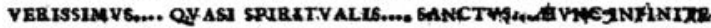
PERDVCAT AD ATRIA vite. AMQ.

-15 -

1183

Consecratio de la iglesia de Sahagún. ${ }^{40}$

A. SAHAGÚN, Monasterio. Piedra de consagración. Sita junto al altar

PUBL, Argaiz, II, p. 548; Flórez, España Sagrada, XVI, p. 220.

HUTUS ALTARIS CONSECRATIO FACTA EST

A DNO FERDINANDO BONE MEMORIE

ASTURICENSI EPISCOPO, IN HONOREN SANCTI BENEDICTL, PRESENTIBUS EPISCOPIS PETRO CIVITATENST ET ADEPHONSO AURIENST: INTRA QUOD SUNT RELIQUIE DE SEPULCRO SANCTE MCARIE, ET SANCTORUM ACARTYRUM FACUNDI ET VICTORICT, ET SANCTI PRUDENTIJ. ADEPHONSO REGE CATHOLICO REGNANTE TOLETO, ET JOANNE ABBATE ECCLESTAM SANCTI FACUNDI ET PRIMITIVI GUBERNANTE, ANNO DOMINI MCLXXXIII. DDIBUS ARRILIS.

$-16-$

S. XII

Consecratio de la iglesia del Sahagún. ${ }^{41}$

A. SAHAGÚN, Monasterio, iglesia. 221.

PUBL: Sandoval, fol. 74; Flórez, España Sagrada, XVI, p.

CUBERNANTE DOMNO JOANNE HANC ABBATIAM SANCTORUM FACUNDI, ET PRMITIVT, CONSECRATA FUIT ECCLESIA HUJUS COENOBII A DOMNO FERDINANDO BONE MEMORLE ASTURIENSI EPISCOPO. CUIUS CORPUS IN EADEM ECCLESIA gEPULTUM EST.

40 Esta Consecratio del monasterio de Sahagún es incluida por el Padre Flórez por ser obra del obispo Fernando II de Astorga. Es por ello que nosotros también la incorporamos a nuestro apéndice.

41 Sucede lo mismo que en el caso anterior. Cf. nota 40. 
$-17-$

1265

Epitaphium sepulcrale del Obispo Pedro V.

A. ASTORGA, Iglesia catedral, capilla de los obispos.

B. Códice 37 de la Iglesia de Astorga, fol. 259; 2a parte de la Nueva Planta de la Iglesia por Antonio de Quiroga y Losada.

PUBL: Canónigo Joseph Antonio Molina; Flórez, España Sagrada, XVI, p. 238. (273)

\section{HIC REQVIESCIT FAMVLVS DEI PETRVS FERNANDI ASTORICENSIS EPISCOPUS QVI ECCLESIAM ISTAM CONSVMMAVIT ET CONSECRAVIT. OVI ETIAM REDIENS DE EXERCITV DE GRANATA OBITT APVD ALBALA PROPE BENQVERENTIAM. VII. IDVS IVNII. ET SEPVLTVS FVIT XIV. KAL. IVLII. ANNO D. M. CC. LXV. ORATE PRO EO}

$$
\text { P. N. A. M. (*) }
$$

$-18-$

1422

Epitaphium sepulcrale del Cardenal Pedro de Fonseca.

A. ROMA, Vaticano, llevado allí a sepultar.

PUBL: Flórez, España Sagrada, XVI, pp. 268-269.

Ortus in Hesperiis Presul dignissimus oris Fonseca de prole Petrus, lax, glaria magne Sanguinis, of patrii superexaltator bonaris Hic jacet: à Sacro titulum Michaele recepit Cardineum: cujus sapientia claruit altas In laudes: sensati animi mirabilis iste DoEtor erat, Divina colens, of amator bonesti, Mente pius, recti prwdens, moderat or oqui. Venit amara dies, quo dira syncopa mortis Heu ! Patrem bune rapuit, Domini labentibus anmis Mille quadringentis bis denis atque duobus, Dum vicena dies Augusti panderet astra. Spiritus in Calo tecum, Sacer Angele, vivat.
BIBLIOGRAFÍA

AA. VV. 2006. El Padre Flórez: tres siglos después. Actas del Congreso Internacional. Burgos 23 al 26 de septiembre de 2002. Burgos: Diputación Provincial de Burgos.

Borreguero Beltrán, C. 2006. «El gigantesco legado del Padre Flórez», en: AA.VV. El Padre Flórez: tres siglos después: Actas del Congreso Internacional: 9-20. Burgos: Diputación Provincia de Burgos.

Campos y Fernández de Sevilla, J. F. 2000. «El Padre Enrique Flórez y la España Sagrada», en E. Flórez, España Sagrada: clave geográfica: 52-60, ed. Rafael Lazcano. Madrid: Editorial Agustiniana.

Favreau, R. 1979. Les inscriptions médiévales. Turnhout: Brepols.

Fernández Flórez, J. A. 2006. "Algunas huellas del "Proyecto de una diplomática española"», en: AA.VV. El Padre Flórez: tres siglos después: Actas del Congreso Internacional: 229-251. Burgos: Diputación Provincial de Burgos.

Flórez, E. 1747. España Sagrada. Theatro geographico-histórico de la Iglesia de España. Origen divisiones y términos de todas sus provincias. Antigüedad traslaciones y estado antiguo y presente de sus sillas en todos los dominios de España y Portugal. Madrid: Oficina de Antonio Marín.

Flórez, E. 1762. España Sagrada theatro geographico-historico de la iglesia de España. Origen, divisiones y limites de todas sus provincias. Antigüedad, translaciones, y estado antiguo y presente de sus Sillas, con varias disertaciones críticas. Tomo XVI. De la santa Iglesia de Astorga en su estado antiguo y presente: (Razón de este libro), Madrid: Imprenta de D. Gabriel Ramírez.

García Lobo, V. 1991. Los medios de comunicación social en la Edad Media. La comunicación publicitaria. León: Universidad de León.

García Lobo, V. 2001. "La epigrafía medieval. Cuestiones de método», en: M. Ruíz Trapero, Centenario de la cátedra de "Epigrafía y Numismática" Universidad Complutense de Madrid 1990/012000-01: 75-120, Madrid: Universidad Complutense de Madrid.

García Lobo, V. y Martín López, Mạ. E. 1995. De epigrafía medieval. Introducción y álbum. León: Universidad de León.

Martín López, Mạ. E. 2007. "Centros escriptorios epigráficos de la provincia de Palencia», en: AA.VV. De Litteris, manuscriptis, inscriptionibus. Festcshrift zum 65. Geburtstag von Walter Koch, herausgegeben von Theo Kölzer, Franz-Albrecht Bornschlegel, Christian Frield, Georg Vogeler: 203-227. Wien: Böhlau.

Peset, M. 2006. "Enrique Flórez y las universidades», en: AA.VV. EI Padre Flórez: tres siglos después: Actas del Congreso Internacional: 113-124. Burgos: Diputación Provincial de Burgos.

Quintana Prieto, A. 1972. "Astorga, Diócesis de», en Diccionario de Historia Eclesiástica de España: 148. Madrid: Consejo Superior de Investigaciones Científicas.

Rodríguez Suárez, N. 2010. Ambrosio de Morales y la Epigrafía Medieval. León: Corpus Inscriptionum Hispaniae Mediaevalium. 